\section{Gesund sein ist für jeden anders}

\author{
KONZEPTE UND THEORIEN VON GESUNDHEIT Zunehmend \\ mehr Ergotherapeuten arbeiten im Bereich der Gesundheits- \\ förderung. Was sie dabei fördern, ist allerdings nicht so klar, \\ wie es zunächst scheint. Denn jeder Mensch hat eine andere \\ Vorstellung von Gesundheit - abhängig beispielsweise von \\ Alter, Geschlecht und sozialem Status.
}

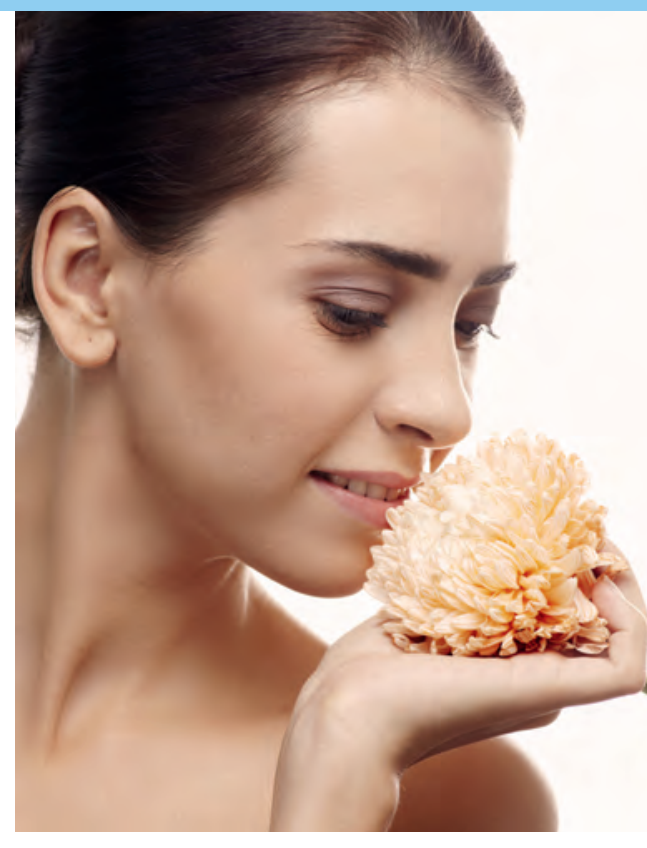

der überwiegende Teil der Menschen hat eigenständige, positive Gesundheitskonzepte, die sich nicht ausschließlich durch die Abwesenheit von Krankheit auszeichnen [1]. Eine Studie aus Großbritannien mit einer repräsentativen Stichprobe konnte beispielsweise zeigen, dass nur 13 Prozent der Bevölkerung die eigene Gesundheit als Abwesenheit von Krankheit versteht. Der überwiegende Teil - 42 Prozent - versteht Gesundheit als Wohlbefinden [5].

Alter und Geschlecht beeinflussen die Gesundheitsvorstellung > Alter und Geschlecht haben - erwartungsgemäß - einen starken Einfluss auf die eigene Gesundheitsvorstellung: Kindern im Vorschulalter fällt es noch schwer, Gesundheit zu definieren. Sie nehmen diese überwiegend als positiven Zustand wahr, der körperliche Aktivität ermöglicht. Ab dem Grundschulalter erkennen die Kinder dann die Reversibilität von Gesundheit und dass Gesundheit und Krankheit gleichzeitig vorhanden sein können („Mein Bein ist krank“) [6]. Bei Jugendlichen wiederum hat das subjektive (Wohl-)Befinden einen hohen Stellenwert, seelisches Gleichgewicht ist besonders den Mädchen wichtig [7]. Auch im Erwachsenenalter assoziieren Frauen Gesundheit eher mit körperlichem und seelischem Wohlbefinden. Für Männer bedeutet sie hingegen funktionale Leistungsfähigkeit und Abwesenheit von Krankheit. Das „männliche“ 
Frauen assoziieren Gesundheit eher mit körperlichem und seelischem Wohlbefinden.

Für Männer bedeutet Gesundheit

überwiegend funktionale Leistungs-

fähigkeit und Abwesenheit von

Krankheit. Das gilt auch für

einige Menschen mit niedrigem

sozioökonomischen Status.

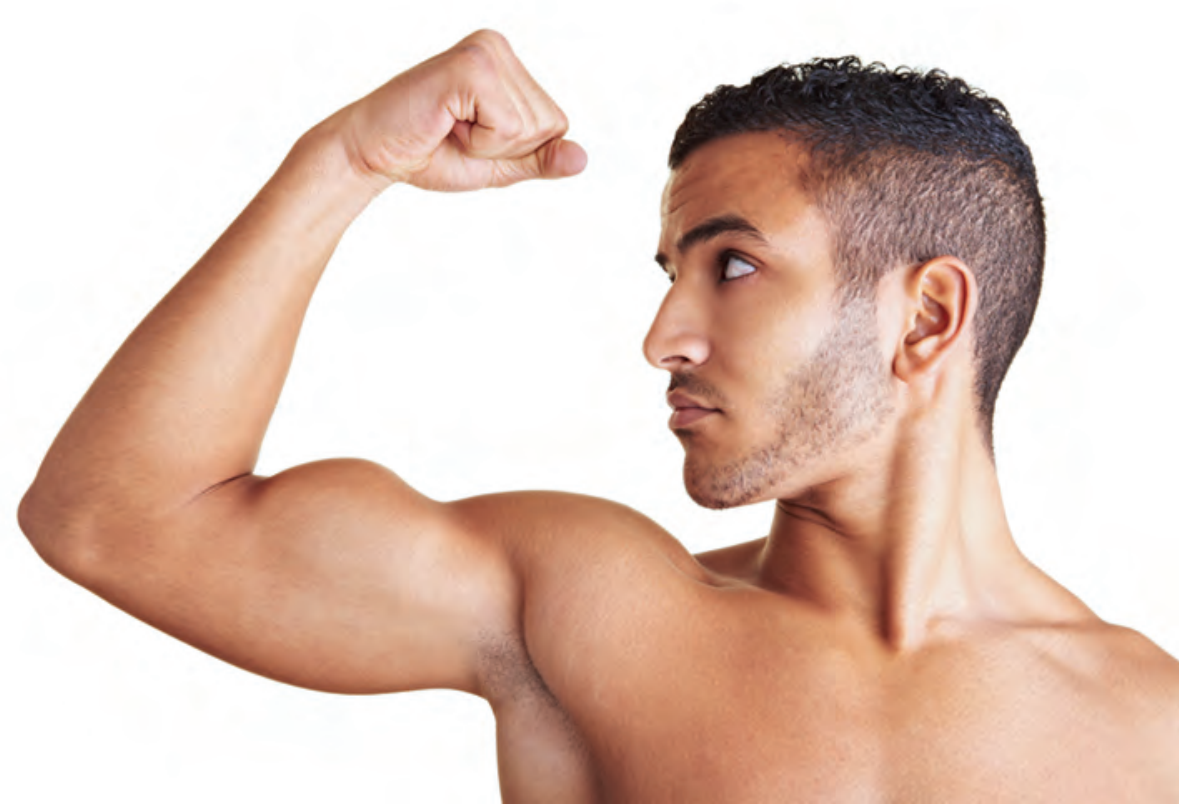

die auf eigenem Verhalten beruhen, sehen sie dagegen prinzipiell als kontrollierbar an.

Ressourcentheoretiker gehen davon aus, dass interne und externe Ressourcen (beispielsweise eine starke Persönlichkeit und vertrauensvolle Beziehungen) die Gesundheit beeinflussen: Je schwächer die Ressourcen eines Einzelnen, desto gefährdeter ist dessen Gesundheitszustand.

Der Meinung von Ausgleichs- und Balancetheoretikern nach ist die Gesundheit zwar durch bestimmte Risiken gefährdet. Sie gehen aber gleichzeitig davon aus, dass es möglich ist, Risiken auszugleichen oder zu kompensieren, etwa durch Entspannung oder Zeit mit der Familie. Zudem sehen sie Gesundheit als herstellbare Balance zwischen körperlichen, psychischen und sozialen Einflüssen an. Dieses Gleichgewicht ist für sie jedoch labil. Es wird durch äußere Einflüsse (zum Beispiel Konflikte am Arbeitsplatz) gefährdet und muss immer wieder neu hergestellt werden.

Für Schicksalstheoretiker sind die Krankheiten, die ihre Gesundheit bedrohen, vorwiegend in biologischen Alterungsprozessen und schicksalhaften Ereignissen begründet. Sie sehen ihre eigenen Einflussmöglichkeiten auf diese Faktoren als sehr gering an [3].

Die eigenen Gesundheitsvorstellungen reflektieren > Man geht davon aus, dass sich die subjektiven Gesundheitsvorstellungen von Ärzten, Therapeuten und Pflegenden unmittelbar auf ihre Interaktion mit den Patienten auswirken sowie auf ihre Entscheidungen und Handlungen. Untersuchungen zeigen, dass deutsche Ärzte und Pflegende Gesundheit eher mit körperlichem und seelischem Wohlbefinden verbinden und sie nicht, wie vielleicht erwartet, rein biomedizinisch als Abwesenheit von Krankheit definieren. Pflegende haben darüber hinaus ein Gesundheitskonzept, das auch Unabhängigkeit und Selbstständigkeit einschließt [4, 11].

Wie kontraproduktiv es sein kann, wenn beispielsweise Ärzte ihre eigenen Gesundheitsvorstellungen und als gesundheitsförderlich definierten Verhaltensweisen nicht reflektieren, zeigen Zitate aus einer dieser Studien: „Was ich für mich selbst als gut empfinde, gebe ich auch an die Patienten weiter“, „Sport empfehle ich gar nicht ... weil ich auch keinen mache“ [11].

Eva Trompetter

\section{$\rightarrow$ Literaturliste: www.thieme-connect.de| products/ergopraxis > „Ausgabe 5/14“}

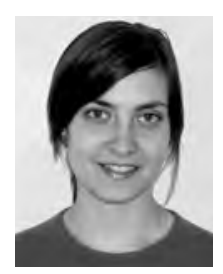

Eva Trompetter hat Public Health studiert und ist wissenschaftliche Mitarbeiterin an der FH Bielefeld. jektiven Gesundheitstheorien ermitteln:

$>$ Ressourcentheorien

$>$ Ausgleichs- und Balancetheorien

$>$ Schicksalstheorien

Risikotheoretiker sehen ihre Gesundheit durch Risiken und Belastungen gefährdet. Externe Risiken wie Schadstoffe aus der Umwelt empfinden sie als schlecht kontrollierbar. Risiken, 